\title{
Analytical evaluation of the fundamental frequency of natural vibrations of the spatial coverage
}

\author{
Mikhail Kirsanov ${ }^{1 *}$ [0000-0002-8588-3871], and Oleg Vorobyev ${ }^{1}$ \\ ${ }^{1}$ National Research University «Moscow Power Engineering Institute», 112250, \\ Krasnokazarmennaya st., Moscow, Russia
}

\begin{abstract}
The scheme of statically definable truss of spatial coverage is proposed. The formula for the dependence of the vibration frequency on the number of panels is derived. The Dunkerley lower bound and the induction method are used to generalize particular solutions to the case of an arbitrary number of panels. The calculation of the forces in the rods by cutting out the nodes and the analytical transformations to obtain the desired dependence are performed in the Maple computer mathematics system. The solution is compared with the numerical one obtained by solving the problem on the eigenvalues of the characteristic matrix for a system with many degrees of freedom. It is shown that the estimation accuracy depends on the number of panels.
\end{abstract}

Keywords: truss, vibration frequency, induction, Maple, spatial coverage.

\section{Introduction}

The problems of natural vibration frequencies of structures are usually solved numerically [1-7]. These solutions are basic for many problems of the dynamics of structures, including the analysis of the seismic resistance of structures [2,8]. Analytical solutions for planar regular systems are also known [9]. The ability to find analytical dependences of frequencies on the number of panels is manifested in regular statically determinate structures. The problems of existence and calculation of such systems were studied in [1012]. The basis for solving the problem of vibration frequency is the problem of finding the rigidity of a structure depending on its order (number of panels). By the method of induction using the operators of the computer mathematics system Maple [13-15], this problem was solved in [16-20].

\section{Materials and methods}

\subsection{Truss scheme and determination of the forces in the rods}

${ }^{*}$ Corresponding author: $\underline{\text { c216@ya.ru }}$ 
The pavement is a rectangular structure with a size $2 n a \times 2 n a$ (Figs. 1,2). The truss rests on the sides on rigid vertical posts [21]. The racks are assumed to be non-deformable and they are included in the calculation of the natural frequency. Support $A$ contains a spherical support, modeled by three mutually perpendicular members. The corner support $B$ contains a cylindrical support of two rods. The total number of elements in the structure is $n_{s}=12 n^{2}+12 n+3$. The truss has a periodic structure. The periodicity cell is a regular pyramid of eight rods of length $a$ at the base and eight inclined braces. The truss is statically determinate. The number of truss rods together with support rods is three times greater than the internal hinges, for the equilibrium condition of which three equations are required.

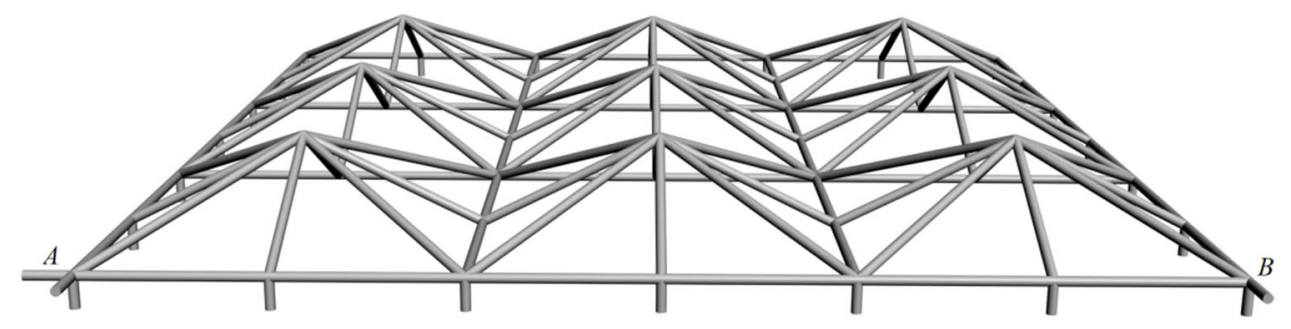

Fig. 1. Truss, $n=3$.

The weight of a truss is distributed over its nodes. Only vertical mass shifts are taken into account. Thus, the number of degrees of freedom of the system is equal to the number of internal nodes $K=(2 n-1)^{2}$.

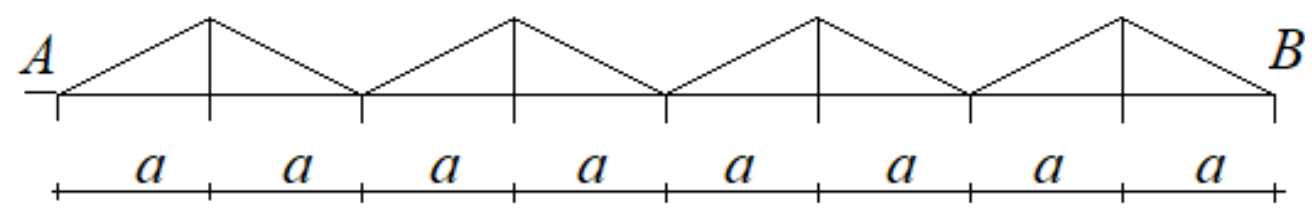

Fig. 2. Truss, $n=4$.

To determine the forces in the rods, a system of equations for the equilibrium of all nodes is drawn up. For this, a coordinate system (Fig. 1) and the coordinates of the nodes are introduced:

$\mathrm{x}_{k}=(i-1) a, \mathrm{y}_{k}=(j-1) a, \mathrm{z}_{k}=h\left(1+(-1)^{i}\right)\left(1+(-1)^{j}\right) / 4, k=i+(j-1)(2 n+1)$, $i, j=1, \ldots, 2 n+1$

Next, a matrix of equilibrium equations is compiled, the elements of which are the direction cosines of the forces. The solution in symbolic form is obtained in the Maple system. This solution is necessary to determine the rigidity of the structure using the Mohr integral.

\subsection{Dunkerley method}

The lower estimate of the first natural frequency [22-24] is calculated from the values of the partial frequencies

$$
\omega_{D}^{-2}=\sum_{p=1}^{K} \omega_{p}^{-2} .
$$


The equation of oscillations of one mass has the form

$$
m \ddot{z}_{p}+D_{p} z_{p}=0, p=1, \ldots, K,
$$

where $z_{p}$ is the vertical coordinate of the moving mass, $\ddot{z}_{p}$ - acceleration. The stiffness coefficient $D_{p}$ is the inverse of the flexibility value $\delta_{p}=1 / D_{p}$. Flexibility depends on the position of the mass on the cover, and is calculated by the Mohr integral. The sum of the Mohr integral does not include the $8 n+3$ forces of the support non-deformable members:

$$
\delta_{p}=1 / D_{p}=\sum_{j=1}^{n_{s}-8 n-3}\left(S_{j}^{(p)}\right)^{2} l_{j} /(E F) .
$$

The following symbols are introduced: $S_{j}^{(p)}$ - the force in the member with the number $j$ from the action of the vertical unit force applied to the node, $l_{j}$ - the length of the member $j$. Each mass has its own stiffness coefficient and its own (partial) frequency. In the case of harmonic oscillations $z_{p}=U_{p} \sin (\omega t+\varphi)$ from (2) follows $\omega_{p}=\sqrt{D_{p} / m}$. Substituting this expression in (1), we get:

$$
\omega_{D}^{-2}=m \sum_{p=1}^{K} \delta_{p}=m \Delta .
$$

Calculating a series of trusses with a sequentially increasing number of panels, we find that the general form of the coefficient does not depend on the number of panels:

$$
\Delta=\left(C_{1} a^{3}+C_{2} c^{3}+C_{3} d^{3}\right) /\left(E F h^{2}\right),
$$

where $c=\sqrt{2 a^{2}+h^{2}}, d=\sqrt{a^{2}+h^{2}}$.

Calculating the common terms of the sequences of coefficients, we obtain the following dependences on the number of panels

$$
\begin{aligned}
& C_{1}=\left(272 n^{6}-384 n^{5}-198 n^{4}+840 n^{3}-357 n^{2}-51 n+13\right) /(270 n), \\
& C_{2}=(2 n-1)\left(8 n^{3}-8 n^{2}+10 n-1\right) / 36, \\
& C_{3}=n(n-1) .
\end{aligned}
$$

Thus, from (2)-(4) we obtain the required formula for the fundamental frequency of the truss

$$
\omega_{D}=h \sqrt{\frac{E F}{m\left(C_{1} a^{3}+C_{2} c^{3}+C_{3} d^{3}\right)}} .
$$

\section{Results and discussion}

Consider a roof truss with the following parameters. The elastic modulus for steel is adopted: $E=2 \cdot 10^{5} \mathrm{MPa}$, the cross-sections of the rods are the same: $\mathrm{F}=40.5 \mathrm{~cm}^{2}$. The nodes contain masses $m=1500 \mathrm{~kg}$. The following dimensions are taken: $a=2 \mathrm{~m}, h=0.3 \mathrm{~m}$. The graph (Fig. 3) shows a comparison of the dependence of the fundamental frequency on the number of panels, calculated by formula (5) by the Dunkerley method, and the numerical solution of the eigenvalue problem, to which the problem of calculating the frequencies of a system with many degrees of freedom is reduced. 


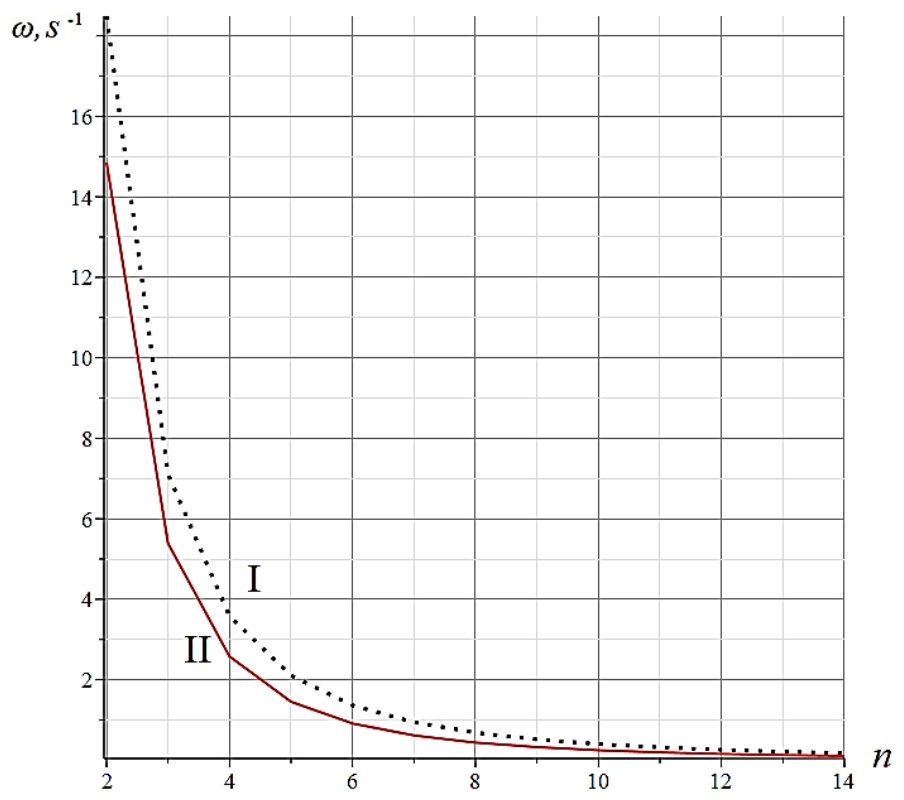

Fig. 3. Frequency dependence on the number of panels: I - the numerical solution $\omega_{1}$ of the eigenvalue problem; II - $\omega_{\mathrm{D}}$, solution (5).

Judging by the graph, the solutions for large values of $n$ approach each other. However, this convergence is absolute, but not relative. The oscillation frequency at these values is low, and the curves seem to almost coincide. However, if you enter a relative error of $\varepsilon=\left(\omega_{1}-\right.$ $\left.\left.\omega_{\mathrm{D}}\right) / \omega_{1}\right)$, the result will be the opposite. The accuracy of Dunkerley's estimate decreases (Fig. 4), asymptotically approaching $40 \%$.

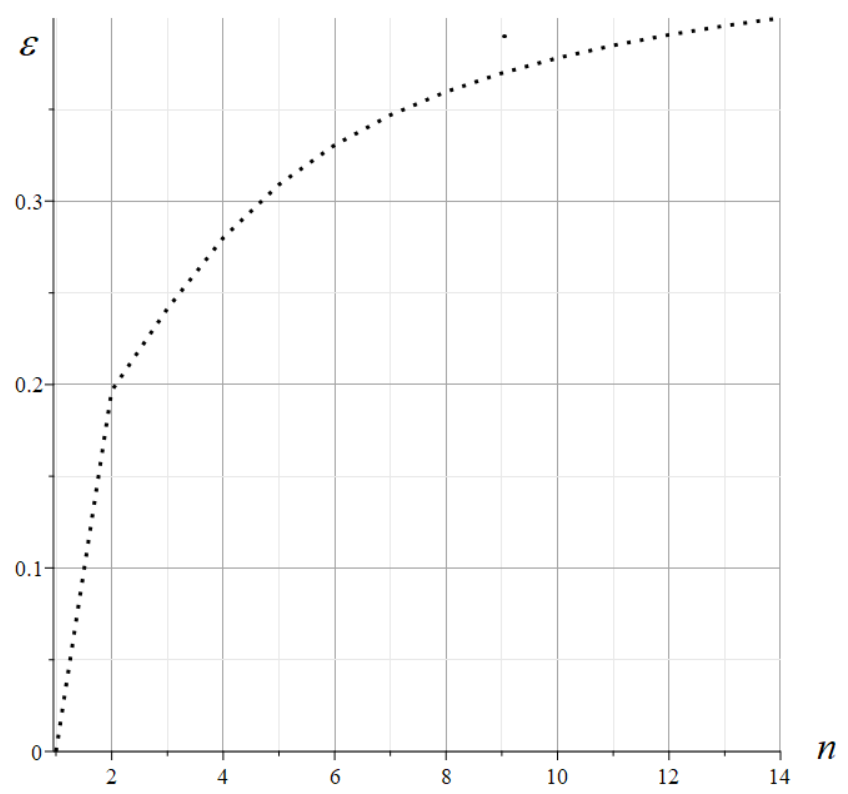

Fig. 4. Relative error of the approximate analytical solution.

Note that Dunkerley's estimate for planar trusses [25] behaves oppositely. With an increase in the number of panels, its accuracy increases. Another feature of solving the natural 
frequency problem for a spatial structure in comparison with the problem for a flat truss consists of a very significant increase in the computation time. This applies both to symbolic transformations in the Maple system, and to numerical ones using the operator Eigenvalues from the Linear Algebra package. If for planar trusses all calculations rarely exceed one or two hours in time, then for a complete solution of the problem for a spatial truss it can take several days.

\section{Conclusions}

Main results of the work are as follows.

1. A dynamic mathematical model of the spatial coverage has been built.

2. The analytical dependence of the fundamental frequency of the truss on the number of panels, dimensions and weight of the structure is obtained.

3. Comparison with the complete numerical solution of the eigenvalue problem showed that the accuracy of the analytical solution decreases with an increase in the number of panels.

\section{Acknowledgements}

The investigation was carried out within the framework of the project «Dynamics of light rod structures of manipulators» with the support of NRU «MPEI» grant for implementation of scientific research programs «Energy», «Electronics, Radio Engineering and IT», and «Industry 4.0, Technologies for Industry and Robotics in 2020-2022».

\section{References}

1. D. Khodzhaev, R. Abdikarimov, N. Vatin, Nonlinear oscillations of a viscoelastic cylindrical panel with concentrated masses, ATEC Web of Conferences, 245 (2018) DOI: $10.1051 /$ matecconf/201824501001

2. N. Vatin, A. Ivanov, Y. Rutman, S. Chernogorskiy, K. Shvetsov, Earthquake engineering optimization of structures by economic criterion, Magazine of Civil Engineering, 8(76), 67-83 (2017) DOI: 10.18720/MCE.76.7

3. J. Chen, W. Zhang, Y. F. Zhang, Equivalent continuum model and nonlinear breathing vibrations of rotating circular truss antenna subjected to thermal excitation, ThinWalled Structures, 157, 107-127 (2020) DOI: 10.1016/j.tws.2020.107127

4. J. Yang, J. Xiong, L. Ma, B. Wang, G. Zhang, L. Wu, Vibration and damping characteristics of hybrid carbon fiber composite pyramidal truss sandwich panels with viscoelastic layers, Composite Structures, 106, 570-580 (2013) DOI: 10.1016/j.compstruct.2013.07.015

5. L. Liang, X. Li, J. Yin, D. Wang, W. Gao, Z. Guo, Vibration characteristics of damping pad floating slab on the long-span steel truss cable-stayed bridge in urban rail transit, Engineering Structures, 191, 92-103 (2019) DOI: 10.1016/j.engstruct.2019.04.032

6. J. Huang, M. Losa, P. Leandri, S. G. Kumar, J. Zhang, Y. Sun, Potential anti-vibration pavements with damping layer: Finite element (FE) modeling, validation, and parametrical studies, Construction and Building Materials, 281, 122550 (2021) DOI: 10.1016/j.conbuildmat.2021.122550 URL: https://linkinghub.elsevier.com/retrieve/pii/ S095006182100310X (date of application: 27.02.2021)

7. L. Cao, J. Liu, Y. Frank Chen, Experimental study on vibration serviceability of steelconcrete composite floor, Structural Engineering and Mechanics, 74(5), 711-722 (2020) DOI: $10.12989 / \mathrm{sem} .2020 .74 .5 .711$

8. A. M. B. Martins, L. M. C. Simões, J. H. J. O. Negrão, Optimization of extradosed 
concrete bridges subjected to seismic action, Computers and Structures, 245 (2021) DOI: 10.1016/j.compstruc.2020.106460

9. M. N.Kirsanov, K. Buka-Vaivade, Analytical expressions of frequencies of small oscillations of a beam truss with an arbitrary number of panels, Structural mechanics and structures, 23(4), 7-14 (2019) URL: vuz.exponenta.ru/PDF/NAUKA/Karina23.pdf (last accessed: 27.02.2021)

10. R. G. Hutchinson, N. A. Fleck, The structural performance of the periodic truss, Journal of the Mechanics and Physics of Solids, 54(4), 756-782 (2006) DOI: 10.1016/j.jmps.2005.10.008

11. R. G. Hutchinson, N. A. Fleck, Microarchitectured cellular solids - The hunt for statically determinate periodic trusses, ZAMM Zeitschrift fur Angewandte Mathematik und Mechanik, 85(9), 607-617 (2005) DOI: 10.1002/zamm.200410208

12. F. W. Zok, R. M. Latture, M. R. Begley, Periodic truss structures, Journal of the Mechanics and Physics of Solids, 96, 184-203 (2016) DOI: 10.1016/j.jmps.2016.07.007

13. B. E. Rapp, Introduction to Maple, Microfluidics: Modelling, Mechanics and Mathematics, 9-20 (2017)

14. K. Zotos, Performance comparison of Maple and Mathematica, Applied Mathematics and Computation, 188(2) 1426-1429 (2007) DOI: 10.1016/j.amc.2006.11.008

15. D. V. Tinkov, Comparative analysis of analytical solutions to the problem of truss structure deflection, Magazine of Civil Engineering, 57(5) (2015) DOI: 10.5862/MCE.57.6

16. M. Kirsanov, Trussed Frames and Arches: Schemes and Formulas (Cambridge Scholars Publishing, UK) URL: www.cambridgescholars.com/product/978-1-52755976-9 (last accessed: 27.02.2021)

17. V. B. Arutyunyan, Analytical calculation of the deflection street bracket for advertising, Postulat, 1 (2019 ) URL: vuz.exponenta.ru/PDF/NAUKA/Arut2019-1.pdf (last accessed: 27.02.2021)

18. A. S. Ilyushin, The formula for calculating the deflection of a compound externally statically indeterminate frame, Structural mechanics and structures, 22(3), 29-38 (2019) URL: elibrary.ru/item.asp?id=41201106 (last accessed: 27.02 .2021 )

19. R. A. Voropay, E. V. Domanov, Analytical solution of the problem of shifting a movable support of a truss of arch type in the Maple system, Postulat, 1 (2019) URL: vuz.exponenta.ru/PDF/NAUKA/VoropDom2019-1.pdf (last accessed: 27.02.2021)

20. A. R. Rakhmatulina, A. A. Smirnova, Analytical calculation and analysis of planar springel truss, Structural mechanics and structures, 17(2), 72-79 (2018) URL: vuz.exponenta.ru/PDF/NAUKA/Rahm-Smirn2018-2.pdf (last accessed: 27.02.2021)

21. M. N. Kirsanov, The Stress-Strain State of a Rectangular Covering Spatial Truss, Construction of Unique Buildings and Structures, 91(6), 9104-9104 (2020) DOI: 10.18720/CUBS.91.4 URL: unistroy.spbstu.ru/article/2020.91.4 (last accessed: 6.03.2021)

22. K. H. Low, Modified Dunkerley formula for eigenfrequencies of beams carrying concentrated masses, International Journal of Mechanical Sciences, 42(7), 1287-1305 (2000) DOI: 10.1016/S0020-7403(99)00049-1

23. C. Levy, An iterative technique based on the Dunkerley method for determining the natural frequencies of vibrating systems, Journal of Sound and Vibration, 150(1), 111118 (1991) DOI: 10.1016/0022-460X(91)90405-9

24. P. G. S. Trainor, A. H. Shah, N. Popplewell, Estimating the fundamental natural frequency of towers by Dunkerley's method, Journal of Sound and Vibration, 109(2) 285-292 (1986) DOI: 10.1016/S0022-460X(86)80009-8

25. M. N. Kirsanov, E. A. Petrichenko, O. V. Vorobev, The formula for the lower estimate of the fundamental frequency of natural vibrations of a truss with an arbitrary number 
of panels, Construction of Unique Buildings and Structures, 94(1), 9403-9403 (2021). DOI: 10.4123/CUBS.94.3. URL: https://unistroy.spbstu.ru/article/2021.94.3 (last accessed: 6.03 .2021 ) 